\title{
Imaging Features of Toxicities by Immune Checkpoint Inhibitors in Cancer Therapy
}

\author{
Gerlig Widmann $^{1} \cdot$ Van Anh Nguyen ${ }^{2} \cdot$ Julian Plaickner $^{1} \cdot$ Werner Jaschke $^{1}$
}

Published online: 11 September 2017

(C) The Author(s) 2017. This article is an open access publication

\begin{abstract}
Purpose of review With the increasing use of immune checkpoint inhibitors in cancer therapy radiographic profiling of frequent and serious immune-related adverse events (irAEs) becomes more relevant. This article reviews imaging features of irAEs induced by the anti-CTLA- 4 and anti-PD-1 antibodies ipilimumab, nivolumab and pembrolizumab.

Recent findings Important radiological manifestations are immune-related colitis, hepatitis, pancreatitis, hypophysitis, pneumonitis, arthritis and sarcoid-like lymphadenopathy. Typical imaging features are summarized and compared with other relevant differential diagnoses.
\end{abstract}

This article is an english version with extended reference list of a german article entitled „Unerwünschte Wirkungen der Immuntherapie: Klinik, Radiologische und nuklearmedizinische Befunde“ published in Der Radiologe 2017, DOI: 10.1007/s00117017-0285-0.

This article is part of the Topical collection on Oncology.

Gerlig Widmann

gerlig.widmann@i-med.ac.at

Van Anh Nguyen

van.nguyen@i-med.ac.at

Julian Plaickner

julian.plaickner@student.i-med.ac.at

Werner Jaschke

werner.jaschke@i-med.ac.at

1 Department of Radiology, Medical University of Innsbruck, Anichstr. 35, 6020 Innsbruck, Austria

2 Department of Dermatology, Venereology and Allergology, Medical University of Innsbruck, Anichstr.

35, 6020 Innsbruck, Austria
Summary Early diagnosis and appropriate therapeutic decisions are required for a successful treatment of irAEs. In addition to staging and follow-up imaging, identification and monitoring of adverse events becomes an important radiologic aspect in oncologic care.

Keywords Immune checkpoint inhibitors - Immunerelated adverse events $\cdot$ Imaging

\section{Introduction}

The recent introduction of a targeted immunotherapy by a novel class of drugs named immune checkpoint inhibitors (ICPIs) has opened new treatment options for several cancer types, such as metastatic melanoma, non-small cell lung cancer, and renal cell cancer [1]. The basic principle of ICPI therapy is the inhibition of negative regulatory components of the T-cell-mediated immune response against cancer cells [2].

As a result of the impaired self-tolerance due to the loss of T-cell inhibition, ICPIs may cause a unique spectrum of immune-related adverse events (irAEs) [3••, 4]. IrAEs can affect any organ and system and may clinically manifest as colitis, hepatitis, pneumonitis, sarcoid-like lymphadenopathy and hypophysitis, which are detectable by computed tomography (CT) magnetic resonance tomography (MRT), or positron emission tomography/computed tomography (PET-CT) [5•]. Symptoms from ICPIs are frequent and may present as serious and life-threatening events, which require timely patient management and adequate therapeutic decisions [6]. Thus, in addition to staging and monitoring of oncologic treatment-using immune-related tumour response criteria (irRC)—radiologists will be in charge for diagnosis and monitoring of 
immune-related toxicities in a growing population of cancer patients receiving immunotherapeutic agents [7-9].

The aim of this article was to review the most relevant imaging features of irAEs induced by ipilimumab, nivolumab and pembrolizumab. Typical radiographic findings were summarized, supported by pictorial examples from our institution and compared with other possible differential diagnoses.

\section{Background}

Ipilimumab was the first ICPI approved in 2011 for treatment of metastatic melanoma. It is a monoclonal antibody that blocks cytotoxic T-lymphocyte-associated antigen 4 (CTLA-4), a checkpoint receptor on T-cells that ligates to B7 molecules (CD80 and CD86) on antigen-presenting cells [2]. A second class of ICPIs are nivolumab and pembrolizumab, which are monoclonal antibodies that block the interaction between another checkpoint receptor on T-cells called cell death protein 1 (PD-1) and its ligands PD-L1 and PD-L2 on antigen-presenting cells and tumour cells [10]. Blocking of CTLA-4 and PD-1 mediated pathways results in activation and proliferation of effector T-cells and simultaneous depletion of regulatory $\mathrm{T}$-cells $[1,2]$. These mechanisms are important stimulators of the cell-mediated cytotoxic effect of the immune system against tumour cells and inhibitors of tumoural immune resistance [2, 11].

The incidence of side-effects of ICPIs is high and may reach up to $80-90 \%$ of patients [12]. Symptomatic irAEs may present with a broad clinical spectrum which can be graded according to Common Terminology Criteria for Adverse Events (CTCAE) as grade 1 (mild), grade 2 (moderate), grade 3 (severe) and grade 4 (life threatening) [13]. A recent review on published toxicity data from phase II and III studies summarized incidences of grade 3 and 4 irAEs of 20-30\% for ipilimumab, 10-15\% for nivolumab and pembrolizumab and $55 \%$ for the combination therapy of ipilimumab and nivolumab [3••]. Toxicities due to ipilimumab are dose dependent and show higher rates of gastrointestinal, dermatologic and endocrine irAEs [3••, 14]. In contrast, toxicities of nivolumab and pembrolizumab are dose independent and associated with higher rates of hepatologic and pulmonary irAEs [3••, 14]. The onset of irAEs after the initiation of ipilimumab may show a temporal association: (a) skin manifestations after 2-3 weeks (1st dose), (b) colitis after 5-10 weeks (2nd dose), (c) hepatitis after 12-16 weeks (3rd dose), (d) endocrine dysfunctions after 9 weeks (4th dose) and (e) pneumonitis after 8-14 weeks [14]. Some patients may simultaneously have more than one irAE [15]. Management of irAEs is based on clinical judgement and follows the CTCAE guidelines [3••, 14]. As a general strategy, ICPIs are interrupted in grade 2 toxicities and resumed when symptoms decrease below grade 1 . For grade 3 and 4 high dose glucocorticoids are given, with additional immunosuppressive agents if symptoms cannot be controlled, and ICPIs are permanently discontinued in patients with grade 4 toxicities. A more detailed description of treatment options would go beyond the scope of this review.

Radiologic manifestations of irAEs may include colitis, hepatitis, pancreatitis, hypophysitis, thyroiditis, pneumonitis, arthritis, sarcoid-like lymphadenopathy and inflammatory changes in the soft tissues (myositis, fasciitis and retroperitoneal fat haziness) [5•, 8, 16•]. Published incidences of radiologic abnormalities obtained from retrospective institutional reviews of patients treated with ipilimumab for metastatic melanoma may amount to $31 \%$ [5•, 16•]. An association between incidence of irAEs and clinical response is suggested [5•]. In scans following treatment of irAEs up to $90 \%$ of imaging findings have resolved [5•, 16•].

\section{Colitis}

Besides dermatologic manifestation such as pruritus and rash, gastrointestinal toxicities have been identified as the most frequent irAEs [4]. Diarrhoea and colitis typically occur 5 weeks after onset of therapy and show a prevalence of $36-38 \%$ and $8-10 \%$ for ipilimumab and $8-20 \%$ and 1-3\% for nivolumab and prembolizumab, respectively [14]. Grade 3 and 4 colitis are seen in 7-9\% of patients receiving ipilimumab and in approximately $1-2 \%$ for the group with nivolumab and prembolizumab [3••, 14]. Serious and life-threatening events may result from significant bloody diarrhoea and intestinal perforation $[4,17,18]$.

In a retrospective case-series reporting 16 patients with ipilimumab associated colitis with available CT evaluation, Kim et al. [17] found mesenteric vessel engorgement in $83 \%$, bowel wall thickening in $75 \%$, and fluid-filled colonic distension in $25 \%$. Pericolic fat stranding was seen in $16 \%$ of patients in the case-series reported by Barina et al. [19]. In both publications there were no patients with pneumatosis or pneumoperitoneum [17, 19]. Immune-related colitis may present in three different patterns (see Table 1 and example in Fig. 1): (a) diffuse colitis, (b) segmental colitis associated with diverticulitis and (c) isolated rectosigmoid colitis without diverticulosis [19]. Colonic wall thickness was greater and pericolic fat stranding was more frequently observed in the segmental colitis associated with diverticulitis pattern than in the diffuse colitis pattern [17]. Clinical symptoms with mixed watery and bloody diarrhoea and cramping pain were more severe in the segmental colitis associated with diverticulitis pattern, whereas diffuse colitis and isolated recto-sigmoid colitis without diverticulosis pattern seemed less symptomatic with a predominance of watery diarrhoea [17, 19]. Presence of an ipilimumab 
induced ileitis without colits is extremely rare and has been described in one case report [20].

Important differential diagnoses of immune mediated colitis are Crohn's Disease, ulcerative colitis, infectious and pseudomembranous colitis (see Table 1). Crohn's disease predominately involves the terminal ileum and has a patchy transmural distribution, ulcerative colitis typically affects the colon with increasing intensity distally, infectious colitis is most commonly limited to the right colon and pseudomembranous colitis shows a marked circumferential or eccentric wall thickening throughout the colon $[21,22]$.

\section{Hepatitis}

Immune-related hepatotoxic effects leading to elevation of hepatic transaminases with or without bilirubin may be observed in 1-10\% of patients on monotherapy but up to $30 \%$ of patients receiving combination therapy of nivolumab plus ipilimumab [3・•, 14]. Grade 3 toxicity with elevated hepatic transaminases 3-5 times the upper limit of normal has been recorded in around 1-2\% of patients receiving monotherapy and $14 \%$ of patients treated with the combination [3・0]. Patients are usually asymptomatic but may sometimes present with fever, fatigue, jaundice and changes of stool colour [22].

On CT and MRT a manifest immune-related hepatitis may present with hepatomegaly, periportal oedema, and attenuated liver parenchyma compared with the baseline CT, periportal T2-hyperintensity on MRT, and enlarged periportal lymph nodes (see Table 1; Fig. 2) [7, 16•, 23]. On ultrasound (US), prominent periportal echogenicity and gallbladder wall oedema were described [22, 23]. In a caseseries of 6 patients with ipilimumab associated hepatitis, Kim et al. [23] reported that a decreased attenuation may obscure hepatic metastases and that new geographic areas of low-attenuation may mimic metastases. On follow-up imaging after glucocorticoid therapy hepatomegaly and periportal lymphadenopathy usually resolve [23].

Imaging findings are non-specific and may be similar to that of viral and alcohol induced acute hepatitis, and idiopathic autoimmune hepatitis [24]. Further, extrahepatic cholestasis and new onset of metastatic liver disease have to be ruled out [23].

\section{Pancreatitis}

ICPI related pancreatitis is rare with an incidence of $<1 \%$ [16•]. It is associated with increase in serum amylase and lipase, may be clinically asymptomatic or present with upper abdominal pain [14].
On CT and MRT, pancreatic enlargement, decrease in attenuation and surrounding fat stranding can be seen (see Table 1; Fig. 3) [16•]. PET-CT may show intense fluor deoxy glucose (FDG) FDG uptake in the pancreas [9]. The findings may be difficult to distinguish from immunoglobulin G4-related autoimmune pancreatitis $[9,25]$. However, the latter may include focal forms, may show a typical loss of the normal fatty lobulations described as a 'sausage pancreas' and may include simultaneous findings in multiple other organs (such as biliary, salivary, aortic and retroperitoneal involvement) [26, 27]. Magnetic resonance cholangiopancreatography (MRCP) may show diffused narrow or segmental stenosis of the main pancreatic duct ('pancreatic duct penetrating sign') without an upstream dilation, strictures of the pancreatic segment of common bile duct, proximal bile duct dilation and gallbladder enlargement $[25,27,28]$. In addition, immunoglobulin G4-related autoimmune pancreatitis often presents with obstructive jaundice and upper abdominal pain [26].

\section{Pneumonitis}

Pulmonary toxicities are rare and typically occur late after about 10 months from the start of ICPI treatment [14]. Symptoms may range from asymptomatic and mild in grade 1 and 2 to cough, hypoxia and life-threatening respiratory failure in grade 3 and 4 , requiring hospitalization and immediate intervention, respectively [6, 14]. Pneumonitis has been more frequently reported in patients treated for advanced lung cancer $(3-5 \%)$ than in patients for malign melanoma $(0-2 \%)$ [3*0]. Prevalence is higher for nivolumab and pembrolizumab and the nivolumab/ipilimumab combined therapy than for ipilimumab monotherapy $[12,29]$. Patients with pre-existing lung disease and who received radiotherapy of lung metastases before ICPI therapy may have an increased risk for developing immune-related pneumonitis [30, 31].

Following the classification according to the American Thoracic Society/European Respiratory Society classification of interstitial pneumonias, Nishino et al. [32] summarized the following imaging findings obtained from 20 patients with PD1-related pneumonitis: a) cryptogenic organizing pneumonia (COP) pattern in $65 \%$, b) nonspecific interstitial pneumonia pattern (NSIP) in $15 \%$, c) hypersensitivity pneumonitis (HP) pattern in $10 \%$ and d) acute interstitial pneumonia/acute respiratory distress syndrome (AIP/ARDS) pattern in 10\%. The lower lungs were more frequently involved than the middle and upper lungs and mixed and multifocal distributions were dominant over peripheral and lower and diffuse distributions [32]. Ground glass opacities were found in all, and reticular opacities and 
Table 1 Typical imaging findings of immune-related adverse events (irAEs) and important differential diagnoses

\begin{tabular}{|c|c|c|}
\hline irAE & Typical imaging findings & Important differential diagnoses \\
\hline Colitis & $\begin{array}{l}\text { Diffuse colitis pattern: diffuse colonic wall thickening, } \\
\text { mucosal hyperenhancement, mesenteric vessel } \\
\text { engorgement } \\
\text { Segmental colitis associated with diverticulosis pattern: } \\
\text { Colonic wall thickening, pericolic fat stranding, mesenteric } \\
\text { vessel engorgement, mucosal hyperenhancement, } \\
\text { diverticula } \\
\text { Isolated recto-sigmoid colitis without diverticulosis pattern: } \\
\text { Colonic wall thickening, mucosal hyperenhancement, } \\
\text { pericolic fat stranding }\end{array}$ & $\begin{array}{l}\text { Crohn's disease: } \\
\text { predominately involves the terminal ileum, patchy } \\
\text { transmural distribution, inflammatory stranding, } \\
\text { submucosal fat, fibrofatty hyperplasia } \\
\text { Ulcerative colitis: } \\
\text { Affects the colon with increasing intensity distally, } \\
\text { Diffuse crypt atrophy, submucosal halo of fat } \\
\text { Infectious colitis: } \\
\text { Most commonly limited to the right colon, wall thickening, } \\
\text { homogeneous enhancement } \\
\text { Pseudomembranous colitis: } \\
\text { Marked circumferential or eccentric colonic wall } \\
\text { thickening }\end{array}$ \\
\hline Hepatitis & $\begin{array}{l}\text { CT, MRT: hepatomegaly, periportal oedema, attenuated } \\
\text { liver parenchyma, periportal lymphadenopathy } \\
\text { US: prominent periportal echogenicity, gallbladder wall } \\
\text { oedema }\end{array}$ & $\begin{array}{l}\text { Viral hepatitis: } \\
\text { Positive viral serology } \\
\text { Acute alcoholic liver disease: } \\
\text { History of alcohol abuse, steatohepatitis, cirrhosis } \\
\text { Idiopathic autoimmune hepatitis: } \\
\text { Cirrhosis, autoantibodies }\end{array}$ \\
\hline Pancreatitis & $\begin{array}{l}\text { CT, MR: pancreatic enlargement, decreased enhancement, } \\
\text { surrounding fat stranding } \\
\text { PET-CT: increased FDG uptake }\end{array}$ & $\begin{array}{l}\text { Immunoglobulin G4-related disease: } \\
\text { Loss of the normal fatty lobulations 'sausage pancreas', } \\
\text { may show focal forms, may include simultaneous findings } \\
\text { in multiple other organs (e.g. biliary, salivary, aortic, } \\
\text { retroperitoneal), } \\
\text { MRCP: diffused narrow or segmental stenosis of the main } \\
\text { pancreatic duct ('pancreatic duct penetrating sign') } \\
\text { without an upstream dilation, may show strictures of the } \\
\text { pancreatic segment of common bile duct, proximal bile } \\
\text { duct dilation, gallbladder enlargement }\end{array}$ \\
\hline Pneumonitis & $\begin{array}{l}\text { Patterns: } \\
\text { Cryptogenic organizing pneumonia }(\mathrm{COP})>\text { non-specific } \\
\text { interstitial pneumonia (NSIP) > hypersensitivity } \\
\text { pneumonitis (HP) and acute interstitial pneumonia/acute } \\
\text { respiratory distress syndrome (AIP/ARDS) } \\
\text { Involvement: } \\
\text { Lower > middle > upper lungs } \\
\text { Distribution: } \\
\text { Mixed and multifocal > peripheral and lower and diffuse } \\
\text { Specific findings: } \\
\text { Ground glass opacities, reticular opacities, consolidations }\end{array}$ & $\begin{array}{l}\text { Bacterial pneumonia: } \\
\text { Consolidations with air-bronchogram, pleural effusion } \\
\text { Drug-induced pneumonitis: } \\
\text { Review of medication history } \\
\text { Radiation pneumonitis: } \\
\text { Involves the lung area that is exposed by a radiation dose } \\
\text { above a threshold of } 30-40 \text { Gray, } \\
\text { Not limited by anatomical borders such as interlobar } \\
\text { fissures and bronchovascular structures, shows ground } \\
\text { glass opacities which may increase in density and } \\
\text { consolidate over time }\end{array}$ \\
\hline Hypophysitis & $\begin{array}{l}\text { Moderate symmetric enlargement of the pituitary, convex } \\
\text { aspect, enlargement of the stalk or infundibulum, } \\
\text { homogeneous contrast enhancement }\end{array}$ & $\begin{array}{l}\text { Pituitary adenoma: } \\
\text { Asymmetric enlargement, heterogeneous contrast } \\
\text { enhancement, loss of pituitary bright spot } \\
\text { Pituitary metastasis: } \\
\text { Melanoma, breast, and lung cancer (rare) } \\
\text { Lymphocytic hypophysitis: } \\
\text { Young women during pregnancy or postpartum period with } \\
\text { headache, visual impairment, and ACTH deficiency }\end{array}$ \\
\hline
\end{tabular}


Table 1 continued

\begin{tabular}{|c|c|c|}
\hline irAE & Typical imaging findings & Important differential diagnoses \\
\hline Arthritis & $\begin{array}{l}\text { US and MRT: proliferative synovitis (hyperaemia and } \\
\text { synovial thickening), joint effusions, sometimes joint } \\
\text { erosions, tenosynovitis, bone marrow edema, myositis } \\
\text { PET-CT: Increased FDG uptake in the synovia of multiple } \\
\text { bilateral joints } \\
\text { Pattern: } \\
\text { Non-specific, large and small joints, may present as } \\
\text { oligoarthritis, additive arthritis, or severe polyarthritis }\end{array}$ & $\begin{array}{l}\text { Rheumatoid arthritis: } \\
\text { Symmetrical small joint involvement, metacarpophalangeal } \\
\text { and proximal interphalangeal joints, rheumatoid factor, } \\
\text { anti-cyclic citrullinated peptide antibodies }\end{array}$ \\
\hline $\begin{array}{l}\text { Sarcoid-like } \\
\text { lymphadenopathy }\end{array}$ & $\begin{array}{l}\text { Symmetric mediastinal and hilar lymph enlargement with } \\
\text { either new lymph nodes or enlargement of pre-existing } \\
\text { nodes }\end{array}$ & $\begin{array}{l}\text { Metastatic lymphadenopathy: } \\
\text { May be asymmetric, may show inhomogeneous } \\
\text { enhancement with natural or treatment related necrosis }\end{array}$ \\
\hline
\end{tabular}
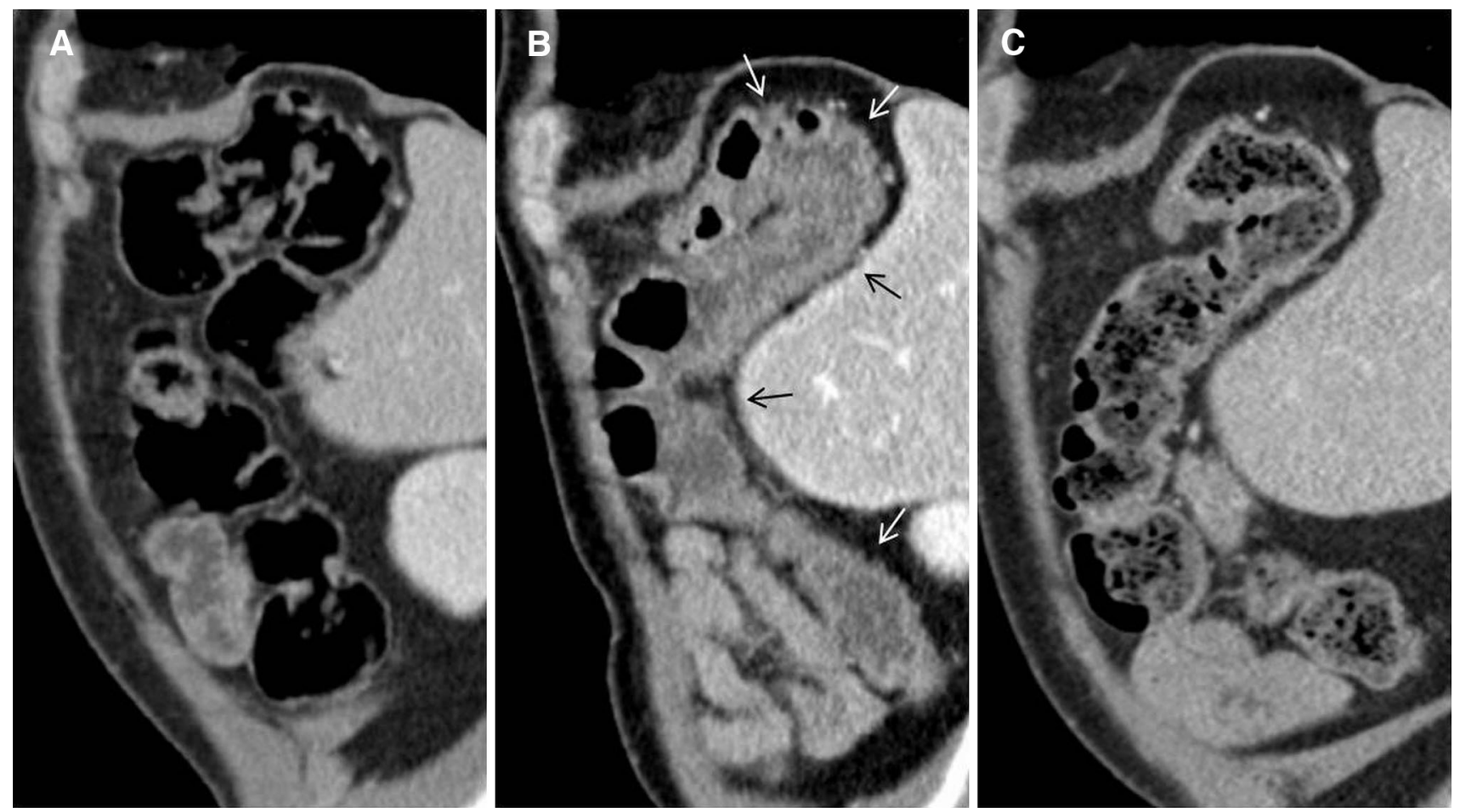

Fig. 1 72-year-old man with colitis grade 2 after 4th dose of ipilimumab. a Normal colon before ipilimumab. b Colitis with diffuse colitis pattern. Colonic wall thickening and mucosal hyperenhancement (arrows). c Improvement after ipilimumab interruption and glucocorticoids

consolidations in most of the cases [32]. Table 1 shows a summary of these findings and an example is given in Fig. 4. A'flare' of clinical and imaging signs of recurrent pneumonitis may occur after taper of glucocorticoid therapy $[32,33]$.

Imaging may help ruling out other pulmonary diseases such as bacterial pneumonia, which typically show asymmetrical consolidations with air-bronchogram and pleural effusion [34]. In addition, resistance to antibiotic treatment, negative sputum, bronchioalveolar lavage and pleural fluid culture may be important clues to support the diagnosis of immune-related pneumonitis [34]. To distinguish pulmonary toxicities from cytotoxic and non-cytotoxic drugs a careful review of the patient's medication history is required [35]. Radiation pneumonitis usually involves the lung area that is exposed by a radiation dose above a threshold of 30-40 Gray and is not limited by anatomical borders such as interlobar fissures and bronchovascular 

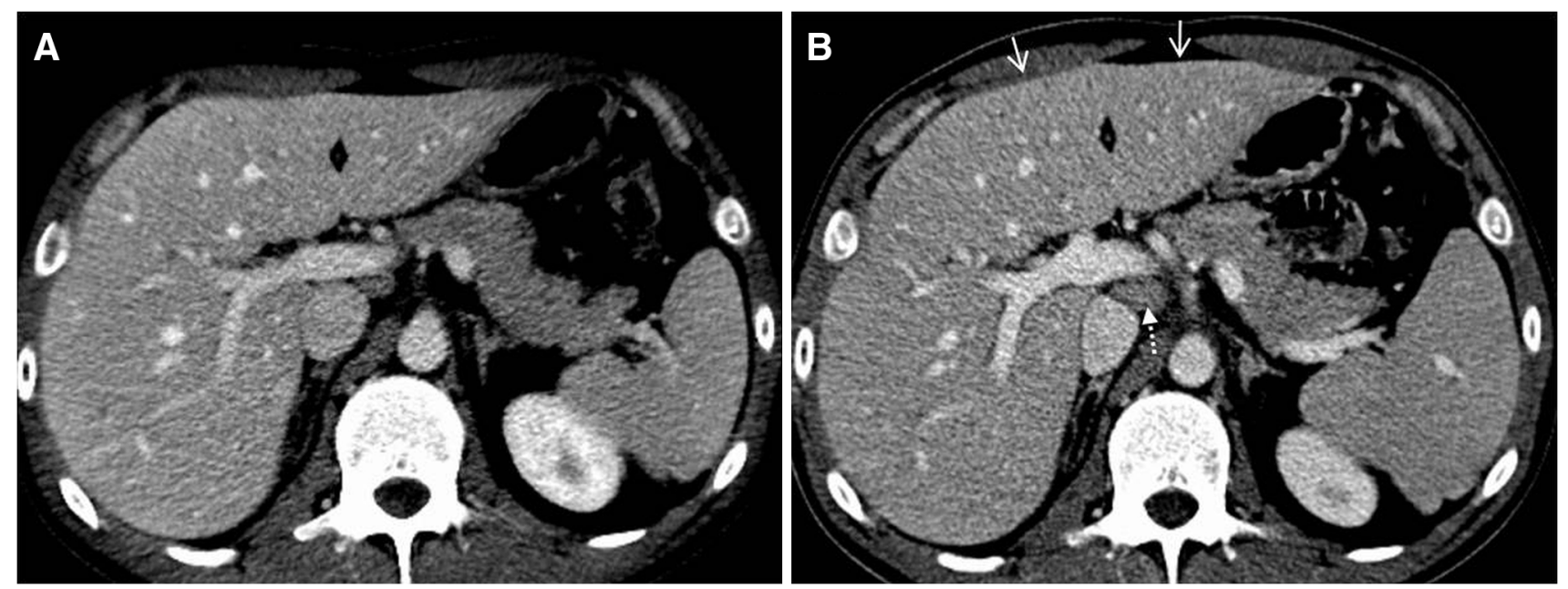

Fig. 2 33-year-old man with hepatitis grade 3 after 3rd dose of nivolumab plus ipilimumab. a Normal sized liver before combination therapy. b Hepatis with convex shaped hepatomegaly (arrows) and periportal lymphadenopathy (dotted arrow)
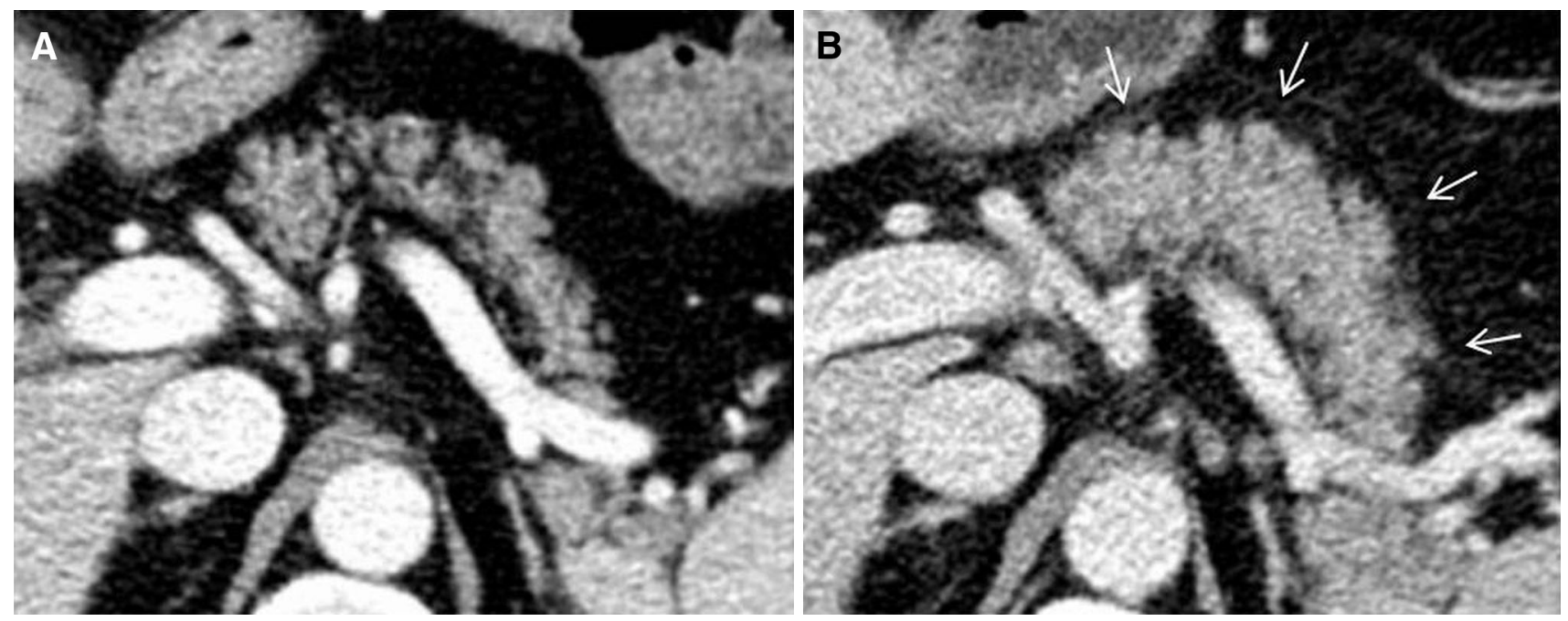

Fig. 3 54-year-old man with pancreatitis after 1st dose of pembrolizumab. a Normal pancreas before pembrolizumab. b Pancreatitis with pancreatic enlargement and fat stranding (arrows)

structures [36]. Onset is after about 6-10 weeks and typical CT findings are ground glass opacities which may increase in density and consolidate over time [36].

\section{Hypophysitis}

Hypophysitis has been reported as a complication of ipilimumab therapy [14]. It is synonymously named as ipilimumab induced hypophysitis (IIH) or ipilimumab associated autoimmune hypophysitis (IAH) [37]. Hypophysitis usually emerges after the third cycle of ipilimumab about 9 weeks from start of treatment and has an incidence of $2-4 \%$ [14]. Higher incidences of $8 \%$ were reported by Larkin et al. [38] for combined nivolumab plus ipilimumab and up to $25 \%$ by Albarel et al. [39] for patients receiving higher dose regimen of ipilimumab $(10 \mathrm{mg} / \mathrm{kg})$. Initial symptoms are headache and fatigue, followed by hypothyroidism, hypogonadism and hypocortisolism, which can be serious and life threatening [14, 37, 39].

In the imaging review by Araujo et al. [37] which included 57 cases of ipilimumab associated hypophysitis, pathologic findings of the pituitary gland were observed in $77 \%$. Typical MRT findings are moderate enlargement of the pituitary, convex aspect, enlargement of the stalk or infundibulum and homogeneous contrast enhancement (see Table 1; Fig. 5) [37, 39]. Heterogeneous contrast enhancement has been reported as an untypical feature [40]. Marlier et al. [41] found an enlargement of the 


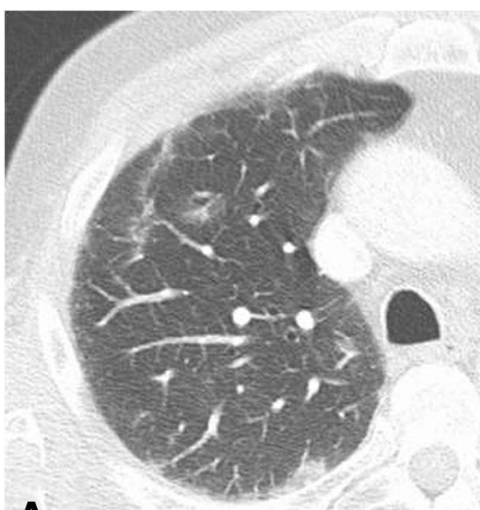

A

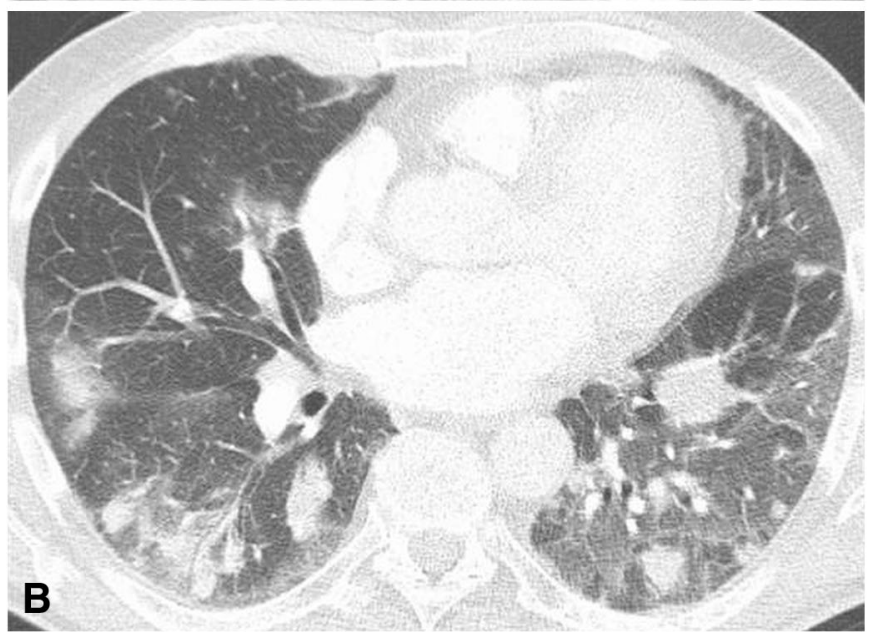

Fig. 4 73-year-old man with pneumonitis grade 3 after 3rd dose of ipilimumab given sequentially after 9 doses of pembrolizumab due to disease progression. $\mathbf{a}$ and $\mathbf{b}$ Pneumonitis with cryptogenic organizing pneumonia pattern. Mixed and multifocal peripheral ground glass

pituitary gland only in the two cases receiving $10 \mathrm{mg} / \mathrm{kg}$ of ipilimumab, whereas the other symptomatic two cases receiving $3 \mathrm{mg} / \mathrm{kg}$ of ipilimumab showed a normal size.

Follow-up MRT after glucocorticoid treatment may demonstrate a decrease in pituitary volume, a change of aspect from convex to concave or in extreme an empty sella [39]. The majority of patients (64-76\%) may show no pituitary function recovery [37, 42]. Immune-related thyroiditis which usually leads to hypothyroidism may occur either isolated or concurrent with hypophysitis and can be detected by increased fluor deoxy glucose (FDG) uptake on PET-CT) [16•].

Important differential diagnoses of ipilimumab associated hypophysitis are pituitary macroadenomas, new onset of brain metastasis and rare pituitary metastasis from melanoma, breast and lung cancer [37]. In contrast to hypophysitis, macroadenomas are usually associated with an asymmetric or focally enlarged pituitary with normal stalk and show a heterogenous enhancement without dural tail [40]. Autoimmune pituitary disease such as

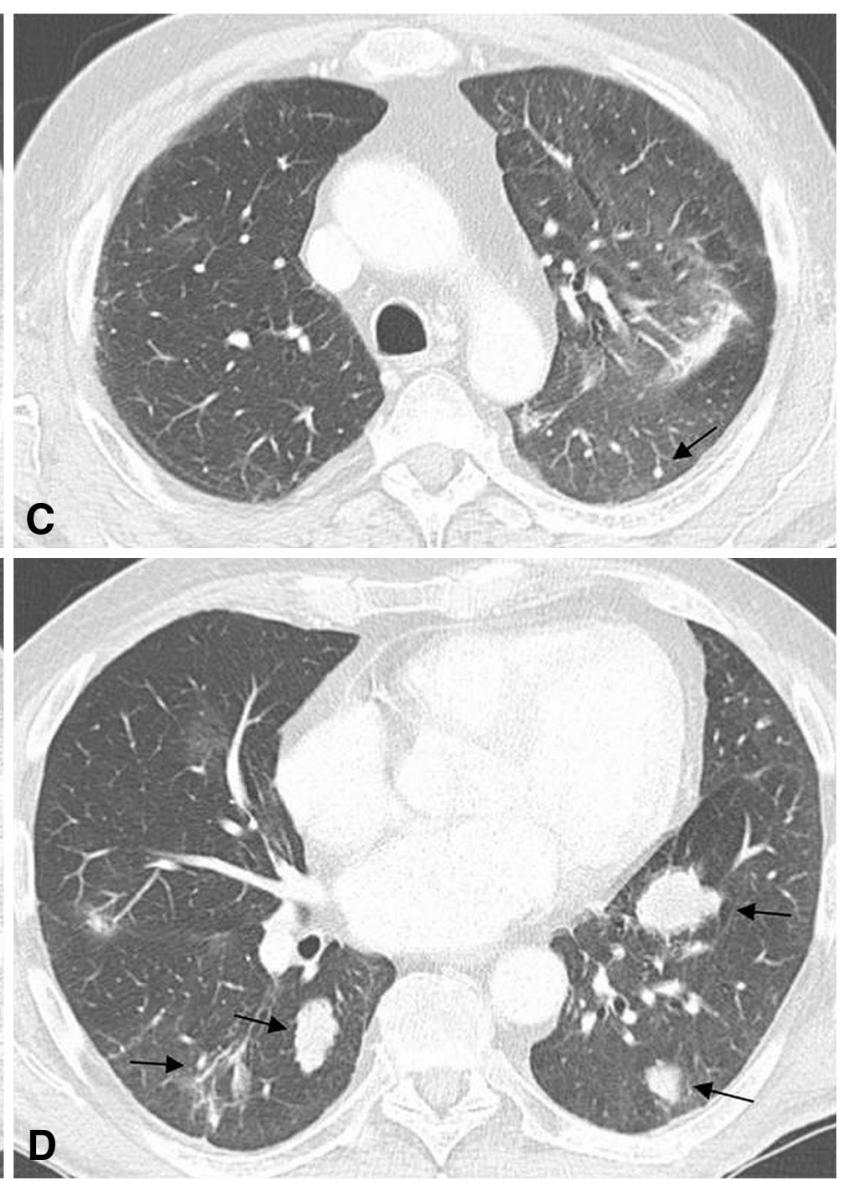

opacities and consolidations. $\mathbf{c}$ and $\mathbf{d}$ Improvement after ipilimumab interruption and glucocorticoids. Arrows show multiple lung metastases

lymphocytic hypophysitis may show identical findings to ipilimumab associated hypophysitis [43, 44]. However, it predominantly affects young women during pregnancy or postpartum period with headache, visual impairment and ACTH deficiency [43].

\section{Arthritis}

Rheumatological side-effects from ICPIs may include arthralgia, myalgia, inflammatory arthritis and myositis [12]. The incidence of arthralgia is around $9-12 \%$ and $6-8 \%$ for patients receiving pembrolizumab and nivolumab, $5 \%$ for patients receiving ipilimumab and $11 \%$ for patients receiving the combination therapy of nivolumab and ipilimumab [3••]. Manifest arthritis is less frequently reported and may affect $2 \%$ of patients on anti-PD-1 inhibitors [3••, 31]. In a case-series of Cappelli et al. [15] including 13 patients with ICPI related rheumatological events, inflammatory arthritis was noted in 9 patients with 

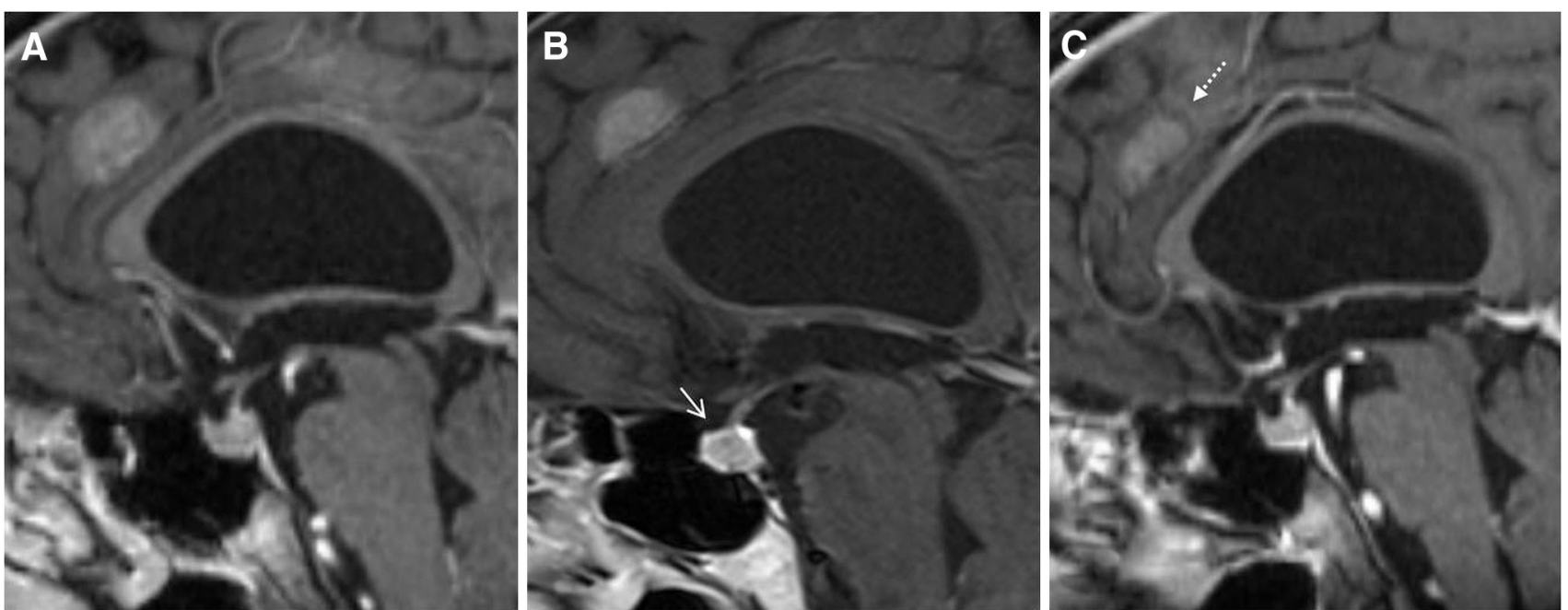

Fig. 5 73-year-old man with hypophysitits grade 3 after 5th dose of ipilimumab. a Normal pituitary gland before ipilimumab. b Hypophysitis with moderate enlargement of the pituitary and

imaging confirmed synovitis and inflammatory synovial fluid in 4 patients. Inflammatory arthritis may affect both large and small joints, and may present as oligoarthritis, additive arthritis or severe polyarthritis [3••, 15, 31].

Imaging findings on US and MRT are proliferative synovitis with typical hyperaemia and synovial thickening, joint effusions and sometimes joint erosions, tenosynovitis, bone marrow edema and myositis (see Table 1; Fig. 6) $[15,45]$. On PET-CT, increased FDG uptake in the synovia of multiple bilateral joints and in muscles may be observed (Fig. 6) $[5 \cdot, 8,9]$. Differentiation from rheumatoid arthritis can be very difficult; however it has been reported that anticyclic citrullinated peptide antibodies and rheumatoid factors are usually absent [15]. Suarez-Almazor et al. [31] postulated two potential pathophysiological explanations for ICPI related arthritis, with one group of patients developing a non-specific arthritis due to up-regulation of the immune system and another group with onset of rheumatoid arthritis bases on a genetic or environmental predisposition.

\section{Sarcoid-Like Lymphadenopathy}

Immune-related sarcoid-like lymphadenopathy has been described as an asymptomatic radiographic finding in around $5-7 \%$ of patients $[5 \cdot, 8,16 \bullet]$. It may present as new onset of symmetric mediastinal and hilar lymph enlargement, with either new lymph nodes or enlargement of preexisting nodes (see Table 1; Fig. 7) [5•]. Tirumani et al. [16•] reported various coexisting pulmonary findings such as bilateral irregular nodular and patchy opacities, or homogeneous contrast enhancement (arrow). c Recovery of pituitary gland to normal size after ipilimumab interruption and glucocorticoids. Dotted arrow shows brain metastasis with partial regression Lymphadenopathy may resolve in most patients $[5 \bullet, 16 \bullet]$.

Differentiation of sarcoid-like lymphadenopathy from metastatic and reactive lymph nodes may be extremely difficult as imaging findings can be unspecific and misleading [8]. Metastatic lymph nodes may show inhomogenous contrast enhancement and necrosis related to natural or treatment related necrosis $\left[5^{\bullet}\right]$.

\section{Conclusions}

Without doubt, with the increasing use of ICPIs in a growing number of tumour types, incidences of immunerelated toxicities will increase. The most important radiologic manifestations of irAE induced by anti-CTLA-4 and anti-PD-1 antibodies may include colitis, hepatitis, pancreatitis, hypophysitis, pneumonitis, arthritis and sarcoidlike lymphadenopathy. Imaging findings of toxic effects from newer ICPIs such as atezolizumab, tremelimumab and pidilizumab may be similar but were not included in this review. Knowledge of typical radiographic features of irAEs is essential to manage early diagnosis and to rule out other relevant differential diagnoses including disease progression. Series and life-threatening events may result from colitis, pneumonitis and hypophysitis. The differentiation of irAEs from autoimmune disorders and immunerelated pneumonitis from infectious and drug-induced findings can be very difficult and requires a close multidisciplinary clinical collaboration. With prompt initiation of therapy according to clinical grades most irAEs can be successfully treated. Identification and monitoring of ground glass and interstitial opacities in 3 of 8 patients. 

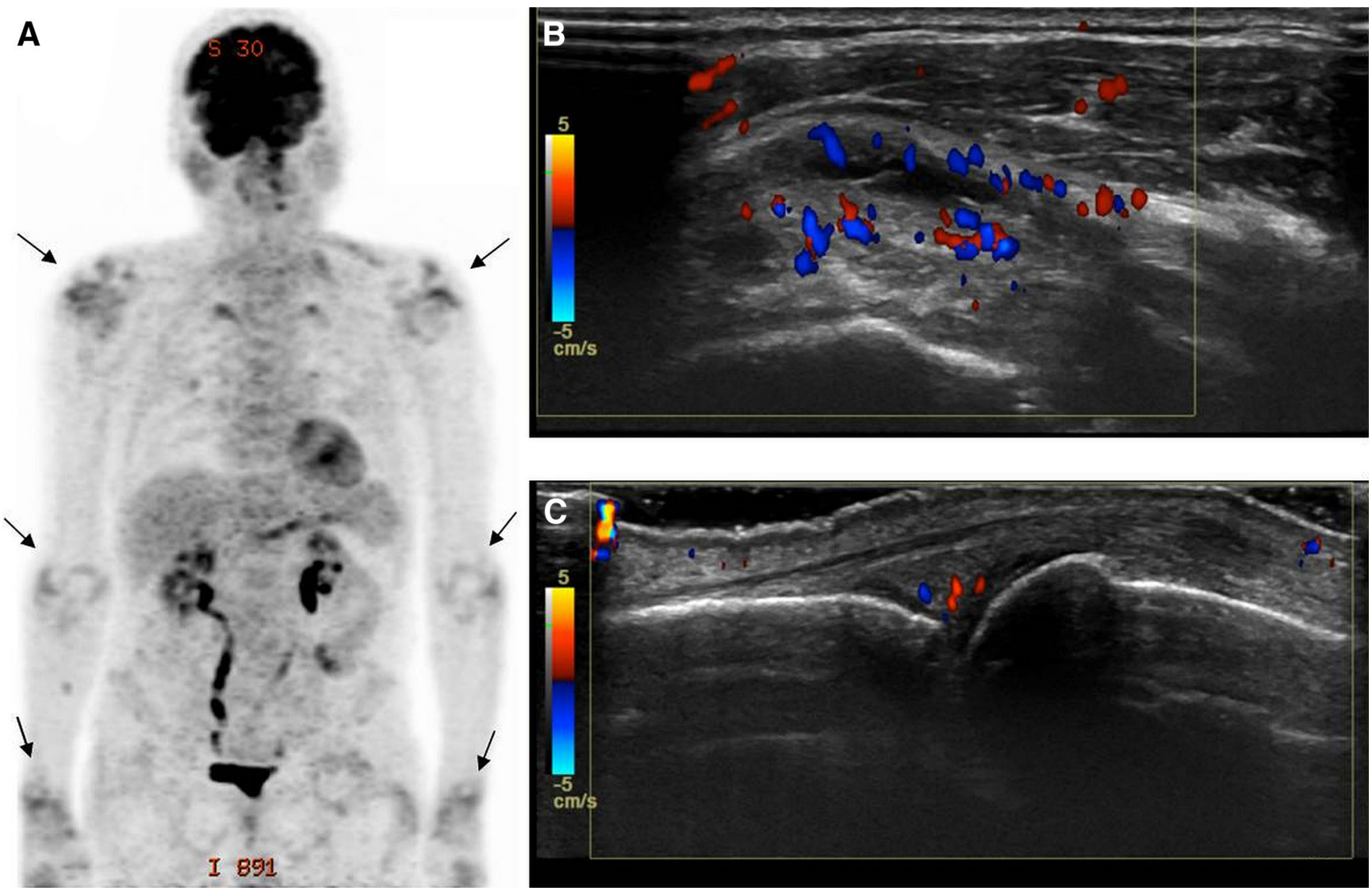

Fig. 6 76-year-old man with arthritis after 21st dose of nivolumab. a PET-CT shows oligoarthritis with increased FDG uptake in the thickened synovial and increased vascularity. c Metacarpophalangeal shoulders, elbows and wrists (arrows). b Shoulder arthritis with
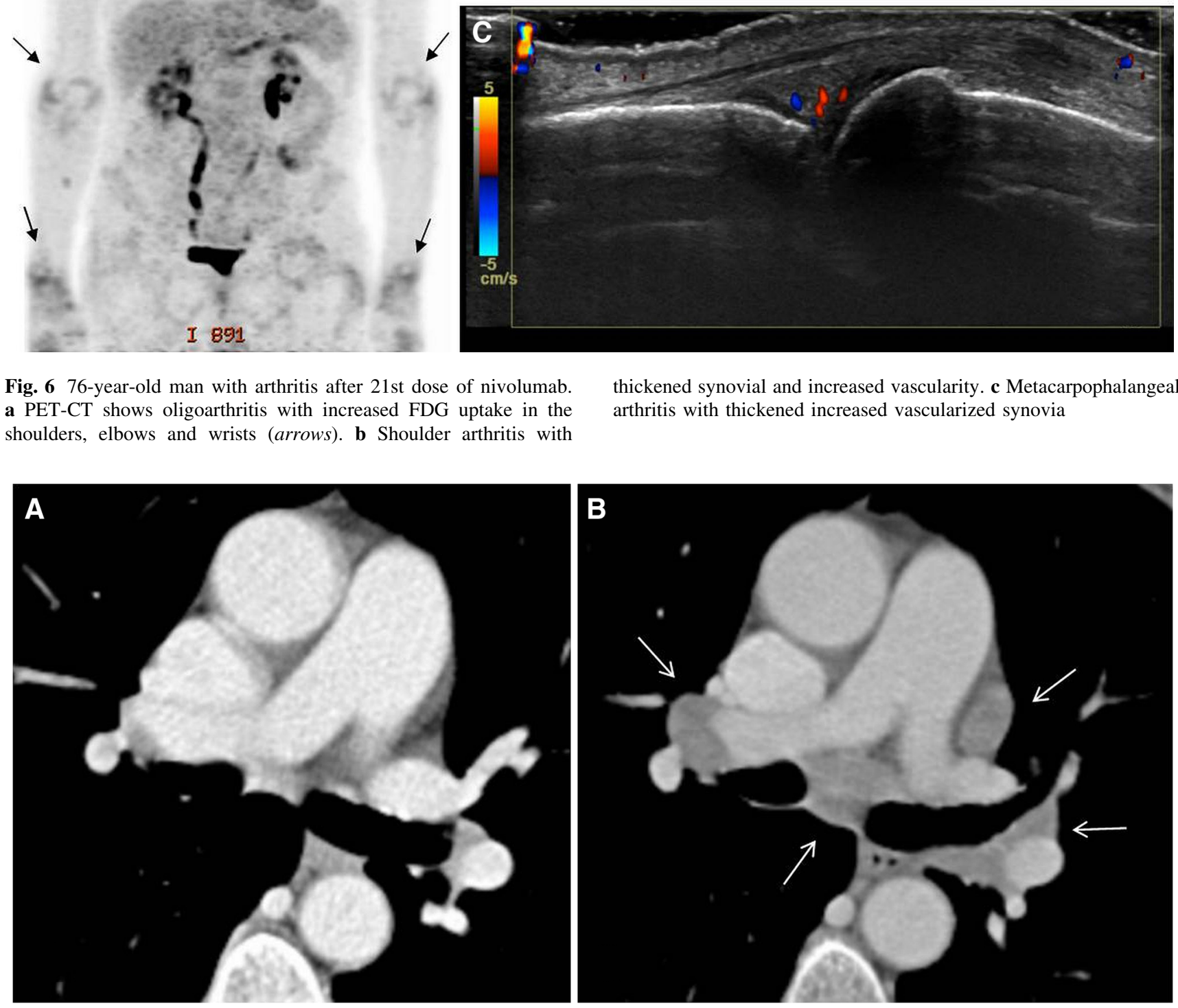

Fig. 7 67-year-old woman with sarcoid lymphadenopathy after 9th dose of pembrolizumab. a Normal mediastinal and hilar lymph nodes before pembrolizumab. b Sarcoid lymphadenopathy (biopsy confirmed) with symmetric hilar and mediastinal enlargement of lymph nodes 
treatment related toxicities increase radiologic responsibility in oncologic care.

Acknowledgements Open access funding provided by University of Innsbruck and Medical University of Innsbruck.

\section{Compliance with Ethical Guidelines}

Conflict of Interest Gerlig Widmann, Van Anh Nguyen, Julian Plaickner and Werner Jaschke each declare no potential conflicts of interest.

Human and Animal Rights and Informed Consent This article does not contain any studies with human or animal subjects performed by any of the authors.

Open Access This article is distributed under the terms of the Creative Commons Attribution 4.0 International License (http:// creativecommons.org/licenses/by/4.0/), which permits unrestricted use, distribution, and reproduction in any medium, provided you give appropriate credit to the original author(s) and the source, provide a link to the Creative Commons license, and indicate if changes were made.

\section{References}

Papers of particular interest, published recently have been highlighted as:

- Of importance

•- Of major importance

1. Dyck L, Mills KH. Immune checkpoints and their inhibition in cancer and infectious diseases. Eur J Immunol. 2017 Apr 09.

2. La-Beck NM, Jean GW, Huynh C, Alzghari SK, Lowe DB. Immune checkpoint inhibitors: new insights and current place in cancer therapy. Pharmacotherapy. 2015;35(10):963-76.

3. - Spain L, Diem S, Larkin J. Management of toxicities of immune checkpoint inhibitors. Cancer Treat Rev. 2016;44:51-60. (Review of immune-related adverse events rates and managment of toxicities.

4. Demlova R, Valik D, Obermannova R, ZdraZilova-Dubska L. The safety of therapeutic monoclonal antibodies: implications for cancer therapy including immuno-checkpoint inhibitors. Physiol Res. 2016;65(Supplementum 4):S455-S62.

5. - Bronstein Y, Ng CS, Hwu P, Hwu WJ. Radiologic manifestations of immune-related adverse events in patients with metastatic melanoma undergoing anti-CTLA-4 antibody therapy. Ajr. 2011;197(6):W992-W1000. )Case review of 20 patients with radiologic manifestations of immune-related adverse events.)

6. Heinzerling L, Goldinger SM. A review of serious adverse effects under treatment with checkpoint inhibitors. Curr Opin Oncol. 2017;29(2):136-44.

7. O'Regan KN, Jagannathan JP, Ramaiya N, Hodi FS. Radiologic aspects of immune-related tumor response criteria and patterns of immune-related adverse events in patients undergoing ipilimumab therapy. AJR. 2011;197(2):W241-6.

8. Braschi-Amirfarzan M, Tirumani SH, Hodi FS Jr, Nishino M. Immune-checkpoint Inhibitors in the era of precision medicine: what radiologists should know. Korean J Radiol. 2017;18(1):42-53.

9. Kwak JJ, Tirumani SH, Van den Abbeele AD, Koo PJ, Jacene HA. Cancer immunotherapy: imaging assessment of novel treatment response patterns and immune-related adverse events. Radiographics. 2015;35(2):424-37.

10. Mahoney KM, Freeman GJ, McDermott DF. The next immunecheckpoint inhibitors: PD-1/PD-L1 blockade in melanoma. Clin Ther. 2015;37(4):764-82.

11. Sweis RF, Luke JJ. Mechanistic and pharmacologic insights on immune checkpoint inhibitors. Pharmacol Res. 2017;18(120):1-9.

12. Kourie HR, Klastersky J. Immune checkpoint inhibitors side effects and management. Immunotherapy. 2016;8(7):799-807.

13. Basch E, Dueck AC, Rogak LJ, Minasian LM, Kelly WK, O'Mara AM, et al. Feasibility assessment of patient reporting of symptomatic adverse events in multicenter cancer clinical trials. JAMA Oncol. $2017 \mathrm{Feb} 16$.

14. Kumar V, Chaudhary N, Garg M, Floudas CS, Soni P, Chandra $\mathrm{AB}$. Current diagnosis and management of immune related adverse events (irAEs) induced by immune checkpoint inhibitor therapy. Front Pharmacol. 2017;8:49.

15. Cappelli LC, Gutierrez AK, Baer AN, Albayda J, Manno RL, Haque $\mathrm{U}$, et al. Inflammatory arthritis and sicca syndrome induced by nivolumab and ipilimumab. Ann Rheum Dis. 2017;76(1):43-50.

16. - Tirumani SH, Ramaiya NH, Keraliya A, Bailey ND, Ott PA, Hodi FS, et al. Radiographic profiling of immune-related adverse events in advanced melanoma patients treated with ipilimumab. Cancer Immunol Res. 2015;3(10):1185-92. )Case review of 46 patients with radiologic manifestations of immune-related adverse events.)

17. Kim KW, Ramaiya NH, Krajewski KM, Shinagare AB, Howard SA, Jagannathan JP, et al. Ipilimumab-associated colitis: CT findings. AJR. 2013;200(5):W468-74.

18. Cheng R, Cooper A, Kench J, Watson G, Bye W, McNeil C, et al. Ipilimumab-induced toxicities and the gastroenterologist. J Gastroenterol Hepatol. 2015;30(4):657-66.

19. Barina AR, Bashir MR, Howard BA, Hanks BA, Salama AK, Jaffe TA. Isolated recto-sigmoid colitis: a new imaging pattern of ipilimumab-associated colitis. Abdom Radiol (NY). 2016;41(2):207-14.

20. Venditti O, De Lisi D, Caricato M, Caputo D, Capolupo GT, Taffon $\mathrm{C}$, et al. Ipilimumab and immune-mediated adverse events: a case report of anti-CTLA4 induced ileitis. BMC Cancer. 2015;01(15):87.

21. Horton KM, Corl FM, Fishman EK. CT evaluation of the colon: inflammatory disease. Radiographics. 2000;20(2):399-418.

22. Cramer P, Bresalier RS. Gastrointestinal and Hepatic Complications of Immune Checkpoint Inhibitors. Curr Gastroenterol Rep. 2017;19(1):3.

23. Kim KW, Ramaiya NH, Krajewski KM, Jagannathan JP, Tirumani SH, Srivastava A, et al. Ipilimumab associated hepatitis: imaging and clinicopathologic findings. Invest New Drugs. 2013;31(4):1071-7.

24. Mortele KJ, Segatto E, Ros PR. The infected liver: radiologicpathologic correlation. Radiographics. 2004;24(4):937-55.

25. Carbognin G, Girardi V, Biasiutti C, Camera L, Manfredi R, Frulloni L, et al. Autoimmune pancreatitis: imaging findings on contrast-enhanced MR, MRCP and dynamic secretin-enhanced MRCP. Radiol Med (Torino). 2009;114(8):1214-31.

26. Hart PA, Zen Y, Chari ST. Recent advances in autoimmune pancreatitis. Gastroenterology. 2015;149(1):39-51.

27. Dillon J, Dart A, Sutherland T. Imaging features of immunoglobulin G4-related disease. J Med Imaging Radiat Oncol. 2016;60(6):707-13.

28. Negrelli R, Manfredi R, Pedrinolla B, Boninsegna E, Ventriglia A, Mehrabi S, et al. Pancreatic duct abnormalities in focal autoimmune pancreatitis: MR/MRCP imaging findings. Eur Radiol. 2015;25(2):359-67. 
29. Abdel-Rahman O, Fouad M. Risk of pneumonitis in cancer patients treated with immune checkpoint inhibitors: a metaanalysis. Ther Adv Respir Dis. 2016;10(3):183-93.

30. Lu CS, Liu JH. Pneumonitis in cancer patients receiving anti-PD1 and radiotherapies: three case reports. Medicine (Baltimore). 2017;96(1):e5747.

31. Suarez-Almazor ME, Kim ST, Abdel-Wahab N, Diab A. Review: immune-related adverse events with use of checkpoint inhibitors for immunotherapy of cancer. Arthritis Rheumatol. 2017;69(4):687-99.

32. Nishino M, Ramaiya NH, Awad MM, Sholl LM, Maattala JA, Taibi M, et al. PD-1 inhibitor-related pneumonitis in advanced cancer patients: radiographic patterns and clinical course. Clin Cancer Res. 2016;22(24):6051-60.

33. Li H, Ma W, Yoneda KY, Moore EH, Zhang Y, Pu LL, et al. Severe nivolumab-induced pneumonitis preceding durable clinical remission in a patient with refractory, metastatic lung squamous cell cancer: a case report. J Hematol Oncol. 2017;10(1):64.

34. Watanabe S, Kimura H, Takato H, Waseda Y, Hara J, Sone T, et al. Severe pneumonitis after nivolumab treatment in a patient with melanoma. Allergol Int. 2016;65(4):487-9.

35. Rossi SE, Erasmus JJ, McAdams HP, Sporn TA, Goodman PC. Pulmonary drug toxicity: radiologic and pathologic manifestations. Radiographics. 2000;20(5):1245-59.

36. Diederich S. Chest CT for suspected pulmonary complications of oncologic therapies: how I review and report. Cancer Imaging. 2016;11(16):7.

37. Araujo PB, Coelho MC, Arruda M, Gadelha MR, Neto LV. Ipilimumab-induced hypophysitis: review of the literature. J Endocrinol Invest. 2015;38(11):1159-66.
38. Larkin J, Hodi FS, Wolchok JD. Combined nivolumab and ipilimumab or monotherapy in untreated melanoma. N Engl J Med. 2015;373(13):1270-1.

39. Albarel F, Gaudy C, Castinetti F, Carre T, Morange I, ConteDevolx B, et al. Long-term follow-up of ipilimumab-induced hypophysitis, a common adverse event of the anti-CTLA-4 antibody in melanoma. Eur J Endocrinol. 2015;172(2):195-204.

40. Chodakiewitz Y, Brown S, Boxerman JL, Brody JM, Rogg JM. Ipilimumab treatment associated pituitary hypophysitis: clinical presentation and imaging diagnosis. Clin Neurol Neurosurg. 2014;125:125-30.

41. Marlier J, Cocquyt V, Brochez L, Van Belle S, Kruse V. Ipilimumab, not just another anti-cancer therapy: hypophysitis as side effect illustrated by four case-reports. Endocrine. 2014;47(3):878-83.

42. Faje AT, Sullivan R, Lawrence D, Tritos NA, Fadden R, Klibanski A, et al. Ipilimumab-induced hypophysitis: a detailed longitudinal analysis in a large cohort of patients with metastatic melanoma. J Clin Endocrinol Metab. 2014;99(11):4078-85.

43. Glezer A, Bronstein MD. Pituitary autoimmune disease: nuances in clinical presentation. Endocrine. 2012;42(1):74-9.

44. Howlett TA, Levy MJ, Robertson IJ. How reliably can autoimmune hypophysitis be diagnosed without pituitary biopsy. Clin Endocrinol (Oxf). 2010;73(1):18-21.

45. Chan MM, Kefford RF, Carlino M, Clements A, Manolios N. Arthritis and tenosynovitis associated with the anti-PD1 antibody pembrolizumab in metastatic melanoma. $J$ Immunother. 2015;38(1):37-9. 